\title{
Contrôle glycémique en réanimation : rêves, désenchantement, espoir
}

\author{
Glycaemic Control in Intensive Care: Dreams, Disappointments and Hopes
}

\author{
J.-C. Preiser \\ Reçu le 2 juin 2017; accepté le 26 juin 2017 \\ (C) SRLF et Lavoisier SAS 2017
}

En réanimation et soins intensifs, les quinze dernières années ont été marquées par un événement majeur : une intervention simple, accessible à tous pour un coût minime a permis de sauver beaucoup plus de vies que tous les outils technologiques et/ou pharmacologiques onéreux et sophistiqués. Il s'agit bien entendu du contrôle glycémique strict par insuline, intervention qui a diminué de moitié la mortalité d'une population de réanimation chirurgicale dans un centre belge [1]. Cette revanche de la simplicité et du bon sens sur la sophistication et la complexité des interventions pharmacologiques onéreuses a suscité un intérêt et un engouement inégalés, révélateurs de nos natures parfois candides et rêveuses. Les cliniciens ont rapidement emboîté le pas et la borne supérieure de la cible glycémique a été abaissée des valeurs traditionnelles inspirées de la diabétologie et du seuil rénal de réabsorption du glucose $(11 \mathrm{mM}$ ou $200 \mathrm{mg} / \mathrm{dl})$ vers un strict 6-8 mM (110-140 mg/dl). Après cette période d'enthousiasme, les études de confirmation multicentriques [2,3] ont été successivement négatives [4]. Le bénéfice impressionnant de l'étude princeps [1] n'a pas été retrouvé, et le contrôle glycémique par insulinothérapie intensive s'est même avéré dangereux en raison des hypoglycémies induites par le traitement. De plus le contrôle strict de la glycémie n'a pu être atteint que péniblement, même dans le cadre des études interventionnelles (proportion de temps passé dans la cible $30-50 \%$ [5]) au prix d'une charge de travail infirmière considérable [6].

Les résultats des études multicentriques ont toutefois permis de mettre en lumière la complexité de la pathogénie de l'hyperglycémie de stress et de manière plus générale d'affiner notre compréhension de la réponse métabolique à l'agression [7]. La comparaison des données de l'étude princeps et des autres études a servi à mettre en lumière les effets délétères induits par l'administration parentérale d'une

\footnotetext{
J.-C. Preiser $(\bowtie)$

Service des soins intensifs, hôpital universitaire Érasme, université libre de Bruxelles, 808 route de Lennik, B-1070 Bruxelles, Belgique

e-mail : jean-charles.preiser@erasme.ulb.ac.be
}

grande quantité de glucose à la phase aiguë de l'agression, pratique courante dans le centre où a été réalisée l'étude princeps $[1,4]$. Les effets toxiques d'apports parentéraux massifs de glucose ont été confirmés ultérieurement par deux études interventionnelles à large échelle initiées également à Louvain, l'une chez l'adulte [8], l'autre chez l'enfant [9].

Indépendamment des apports glucidiques, la définition de la valeur glycémique « optimale » pourrait également varier en fonction du délai depuis l'agression, du type de pathologie aiguë, des antécédents de diabète sucré et de la qualité de son contrôle du diabète. Dès lors, les recommandations actuelles ne peuvent être que peu contraignantes, à savoir une cible glycémique recommandée inférieure à $10 \mathrm{mM}$ [10].

La vague d'enthousiasme déclenchée par l'étude initiale et la vivacité des discussions engendrée par les résultats ultérieurs ont poussé chercheurs et partenaires industriels à collaborer au développement ou à l'amélioration d'outils technologiques performants. Par exemple, les lecteurs de glycémie, les systèmes de monitorage continus, intravasculaires ou interstitiels, et des systèmes de contrôle glycémique à boucle fermée basés sur des algorithmes thérapeutiques dynamiques et individualisés en fonction de l'insulinorésistance instantanée.

Cette évolution, longue d'une quinzaine d'années, nous permet d'entrevoir l'avenir en plusieurs étapes : évaluation des effets cliniques de l'atteinte effective et sans risque d'une cible glycémique définie selon le délai depuis l'agression initiale et le profil du patient (diabétique, cérébrolésé, postopératoire). Ces avancées importantes ne pourront être réalisées que dans le cadre de partenariat entre cliniciens et chercheurs académiques et industriels. Ensuite, l'application et la validation à large échelle de la pertinence de cette approche individualisée. Entre-temps, l'application de l'adage hippocratique « Primum non nocere » trouve bien sa place sous forme d'une cible glycémique générale peu contraignante [10].

Liens d'intérêts : JC Preiser déclare des honoraires de consulting pour Optiscan, Edwards et Maquet. 


\section{Références}

1. van den Berghe G, Wouters P, Weekers F, Verwaest C, Bruyninckx F, Schetz M, Vlasselaers D, Ferdinande P, Lauwers P, Bouillon R, (2001) Intensive insulin therapy in the critically ill patients. N Engl J Med 345: 1359-1367

2. Preiser JC1, Devos P, Ruiz-Santana S, Mélot C, Annane D, Groeneveld J, Iapichino G, Leverve X, Nitenberg G, Singer P, Wernerman J, Joannidis M, Stecher A, Chioléro R, (2009) A prospective randomised multi-centre controlled trial on tight glucose control by intensive insulin therapy in adult intensive care units: the Glucontrol study. Intensive Care Med 35: 1738-1748

3. NICE-SUGAR Study Investigators, Finfer S, Chittock DR, Su SY, Blair D, Foster D, Dhingra V, Bellomo R, Cook D, Dodek P, Henderson WR, Hébert PC, Heritier S, Heyland DK, McArthur C, McDonald E, Mitchell I, Myburgh JA, Norton R, Potter J, Robinson BG, Ronco JJ (2009) Intensive versus conventional glucose control in critically ill patients. N Engl J Med 360: 1283-1297

4. Marik PE, Preiser JC, (2010) Toward understanding tight glycemic control in the ICU: a systematic review and metaanalysis. Chest 137: 544-551
5. Krinsley JS, (2015) Glycemic control in the critically ill: What have we learned since NICE-SUGAR? Hosp Pract 43: 191-197

6. Aragon D, (2006) Evaluation of nursing work effort and perceptions about blood glucose testing in tight glycemic control. Am J Crit Care 15: 370-377

7. Preiser JC, Ichai C, Orban JC, Groeneveld AB, (2014) Metabolic response to the stress of critical illness. Br J Anaesth 113: 945954

8. Casaer MP1, Mesotten D, Hermans G, Wouters PJ, Schetz M, Meyfroidt G, Van Cromphaut S, Ingels C, Meersseman P, Muller J, Vlasselaers D, Debaveye Y, Desmet L, Dubois J, Van Assche A, Vanderheyden S, Wilmer A, Van den Berghe G, (2011) Early versus late parenteral nutrition in critically ill adults. $\mathrm{N}$ Engl $\mathrm{J}$ Med 365: 506-517

9. Fivez T, Kerklaan D, Mesotten D, Verbruggen S, Wouters PJ, Vanhorebeek I, Debaveye Y, Vlasselaers D, Desmet L, Casaer MP, Garcia Guerra G, Hanot J, Joffe A, Tibboel D, Joosten K, Van den Berghe G, (2016) Early versus Late Parenteral Nutrition in Critically Ill Children. N Engl J Med 374: 1111-1122

10. Ichai C, Preiser JC; Société Française d'Anesthésie-Réanimation; Société de Réanimation de langue Française; Experts group, (2010) International recommendations for glucose control in adult non diabetic critically ill patients. Crit Care 14: R166 